\title{
V. - PROPRIÉTÉS DES PROTÉINES
}

\author{
B. GODON et L. PETIT \\ avec la collaboration technique de Alice de PAstors, Éléonore Hudzik et Jeanine Ooghe
}

Laboratoire de Contrôle et de Technologie des Blés, I. N. R. A., 16. rue Nicolas-Fortin, Paris $13^{\mathrm{e}}$

Station de Biochimie et Physicochimie des Céréales, I. N. R. A., C.E.R.D.I.A, 91 - Massy

\section{RÉSUMÉ}

L'étude de l'évolution des protéines au cours des traitements de préstockage et de séchage a été entreprise pour tenter d'expliquer certaines variations de la qualité du maïs. L'état de ces constituants est, en premier lieu, influencé par la température de séchage, mais son action est plus ou moins importante selon le prétraitement subi par les grains : ainsi, une conservation de 45 jours par ventilation d'air refroidi entraîne des modifications plus importantes qu'un échauffement naturel de 6 jours.

\section{INTRODUCTION}

I a qualité du maïs destiné à l'alimentation animale est liée, en grande partie, à l'évolution des constituants biochimiques. Or, les quelques résultats acquis dans le domaine des protéines (Busson et al., I966) n'ont montré aucune modification de la composition en acides aminés à la suite de traitements. On est donc amené à penser que si certaines variations de qualité dépendent des protéines, elles doivent avoir pour origine des transformations de leur structure. Pour le préciser, nous avons étudié l'état de ces substances par des méthodes pouvant fournir des renseignements soit sur leur structure globale, soit, par l'intermédiaire des activités enzymatiques, sur certaines zones particulières.

Les premiers résultats que nous présentons doivent être surtout considérés comme des indices des modifications des protéines cherchant à expliquer certaines variations de la qualité des grains.

\section{MATÉRIEL E'T MÉTHODES}

Les analyses ont été effectuées sur des échantillons représentatifs des lots de mais destinés aux essais zootechniques. Comme cela a été précédemment mentionné, ils ont subi différents traitements de préstockage et de séchage (LASSERAN, I). Afin de dissocier les effets dus aux 
traitements de préstockage « $\mathrm{E}$ » et “ $\mathrm{F}$ » nous avons analysé les échantillons $\mathrm{E}_{0}$ et $\mathrm{F}_{0}$, séchés à température ambiante dans un conditionneur de grain. L'échantillon " témoin de récolte " (TR), séché dans les mêmes conditions, servira de référence pour l'ensemble des résultats.

Les grains de maïs ont été finement broyés dans un appareil Bühler ou Brabender. Nous avons toujours pris soin d'éviter un échauffement trop important en refroidissant le corps de l'appareil à l'aide de glace carbonique, lorsque cela était nécessaire. La teneur en eau a été déterminée selon la méthode de référence mentionnée dans Méthodes analytiques des céréales du CNERNA et le dosage des protéines $(\mathrm{N} \times 5,7)$ a été réalisé par la méthode classique de Kjeldahl.

Sur ces divers échantillons, nous avons examiné :

- la teneur en protéines alcoolosolubles;

- la sensibilité des protéines à la digestion trypsique ;

- l'activité protéolytique,

- la teneur en lysine disponible.

\section{Teneur en proteines alcoolosolubles}

Cette détermination est réalisée à l'aide du solvant utilisé par Busson et al. (Ig66) pour l'étude des acides aminés totaux de la fraction alcoolosoluble : c'est une solution d'alcool à 80 p. Ioo contenant 1,25 p. Ioo d'acide acétique.

L'extraction de cette fraction protéique est effectuée de la façon suivante : $2,5 \mathrm{~g}$ de grains broyés sont introduits dans un tube en verre bouchant émeri et contenant ro ml du solvant alcoolique. Ce tube est disposé sur un agitateur rotatif vertical tournant à $2 \mathrm{t} / \mathrm{minute}$. L'agitation effectuée dans une enceinte à $4^{\circ} \mathrm{C}$ est maintenue pendant $\mathrm{I}_{5} \mathrm{~h}$.

La solution contenant les protéines alcoolosolubles -_- c'est-à-dire les protides de faible poids moléculaire - est récupérée après une centrifugation de 20 minutes à $3000 \mathrm{t} / \mathrm{minutes}$. Les résultats, exprimés en pourcentage du poids des protéines totales, sont obtenus par des dosages d'azote effectués sur la solution recueillie et sur le broyat initial.

\section{2. - Sensibilité des protéines à la digestion trypsique}

La digestion est effectuée au bain-marie à $40^{\circ} \mathrm{C}$, à l'aide d'une solution de trypsine dans un tampon acétate de $\mathrm{pH}_{4,7}$. Cette solution contient $0,32 \mathrm{mg}$ par $\mathrm{ml}$ d'enzyme bovine du commerce (N. B. C.). L'échantillon de maïs broyé est placé dans $25 \mathrm{ml}$ de solution enzymatique en quantité nécessaire pour que la concentration en azote soit de $3,2 \mathrm{mg}$ par $\mathrm{ml}$.

Après 5 heures de digestion, on effectue une précipitation par l'acide trichloracétique à $36 \mathrm{p}$. Ioo, on centrifuge pendant 5 minutes à I $800 \mathrm{t} / \mathrm{minute}$ et l'on filtre. Le pourcentage de protéines digérées est déterminé alors comparativement à la teneur en protéines initiales par dosage de l'azote contenu dans la solution.

\section{3. - Activité protéolytique}

L'activité protéolytique est mesurée par digestion de bactohémoglobine à $40^{\circ} \mathrm{C}$ dans un tampon acétate de $\mathrm{pH}_{4,7}$, suivant la méthode retenue par l'A. A. C. C., et dérivée de la méthode d'Ayre-Anderson. Les résultats sont exprimés en mg d'azote digéré par gramme de substance sèche.

\section{4. - Teneur en lysine disponible}

La détermination de la lysine disponible par voie chimique est effectuée selon la méthode de Carpenter (1960).

\section{RÉSULTATS ET INTERPRÉTATION}

Le tableau I rassemble les résultats obtenus sur l'ensemble des échantillons. La comparaison de ces différentes valeurs à celle du témoin-récolte permet d'observer que : 
- l'importance de la fraction alcoolosoluble est essentiellement influencée par les traitements thermiques : on note une nette diminution de la quantité de protéines de faible poids moléculaire - indice d'une dénaturation - après un séchage à $140^{\circ} \mathrm{C}$ quel que soit le pré-traitement. Par contre, l'influence de l'action thermique à $80^{\circ} \mathrm{C}$ apparaît minime, sauf après une conservation par ventilation d'air refroidi $\left(\mathrm{F}_{80}\right)$.

Nous pouvons aussi remarquer, d'après les résultats de $F_{0}$ et $F_{0}$ que les traitements précédant le séchage n'entraînent pas, par eux-mêmes, de variations vraiment importantes de la solubilité des protéines dans l'alcool.

\section{TABLEAU I}

Résultats analytiques sur les différents échantillons

Valeurs exprimées en pourcentage de la substance brute, de la matière sèche $(\mathrm{MS})$, ou des protéines $(\mathrm{N} \times 5,7)$

\begin{tabular}{|c|c|c|c|c|c|c|}
\hline Échantillons & $\begin{array}{c}\text { Teneur en } \\
\text { eau/s. brute }\end{array}$ & $\left|\begin{array}{c}\text { Teneur en } \\
\text { protéines/MS }\end{array}\right|$ & $\begin{array}{c}\text { Fraction } \\
\text { alcoolosoluble } \\
\% \text { des protéines }\end{array}$ & $\begin{array}{c}\text { Digestion } \\
\text { trypsique } \\
\% \text { des } \\
\text { protéines }\end{array}$ & $\begin{array}{c}\text { Activité } \\
\text { protéolyt. } \\
\text { (mg N/g MS) }\end{array}$ & $\begin{array}{c}\text { Lysine } \\
\text { disponible } \\
\text { /protéines }\end{array}$ \\
\hline $\begin{array}{c}\text { TR } \\
\text { (Témoin } \\
\text { récolte) }\end{array}$ & 14,6 & 9,16 & 24,4 & $2,{ }^{\prime} 4$ & 5,53 & 3,18 \\
\hline $\begin{array}{l}\mathrm{R}_{80} \\
\mathrm{R}_{\mathbf{1 4 0}}\end{array}$ & $\begin{array}{l}14,3 \\
14,1\end{array}$ & $\begin{array}{l}8,75 \\
9,00\end{array}$ & $\begin{array}{l}22,3 \\
11,0\end{array}$ & $\begin{array}{l}3,0 \\
5,0\end{array}$ & $\begin{array}{l}3,39 \\
1,21\end{array}$ & $\begin{array}{l}3,18 \\
2,80\end{array}$ \\
\hline $\begin{array}{l}E_{0} \\
E_{80} \\
E_{140}\end{array}$ & $\begin{array}{l}12,2 \\
13,9 \\
12,0\end{array}$ & $\begin{array}{l}8,95 \\
8,71 \\
8,97\end{array}$ & $\begin{array}{r}24,5 \\
23,3 \\
9,2\end{array}$ & $\begin{array}{l}2,5 \\
2,7 \\
4,7\end{array}$ & $\begin{array}{l}6,12 \\
4,22 \\
2,16\end{array}$ & $\begin{array}{l}3,12 \\
3,10 \\
2,52\end{array}$ \\
\hline $\begin{array}{l}F_{0} \\
F_{80} \\
F_{140}\end{array}$ & $\begin{array}{l}13,2 \\
13,7 \\
13,6\end{array}$ & $\begin{array}{l}9,28 \\
9,15 \\
9,13\end{array}$ & $\begin{array}{l}25,2 \\
17,9 \\
10,1\end{array}$ & $\begin{array}{l}1,9 \\
2,0 \\
3,2\end{array}$ & $\begin{array}{l}9,26 \\
6,76 \\
3,07\end{array}$ & $\begin{array}{l}3,14 \\
3,07 \\
2,61\end{array}$ \\
\hline
\end{tabular}

- la sensibilité des protéines à la digestion trypsique subit une augmentation significative lorsque la température de séchage croît. Mais une diminution relative apparaît si ce traitement est précédé d'un échauffement en cellules et, surtout, d'une conservation par ventilation d'air refroidi. De sorte que, dans ce dernier cas, l'influence du séch.jge à haute température est en grande partie compensée par celle du préstockage.

- un net abaissement de 1'activité protéolytique est mis en évidence déjà après un séchage à $80^{\circ} \mathrm{C}$. Le traitement à $140^{\circ} \mathrm{C}$, directement après la récolte ( $\left.\mathrm{R} \|\right)$, entraîne une activité extrêmement faible. Par contre, les traitements " $\mathrm{E}$ ", et surtout ( $F$ ), produisent une augmentation de l'activité des protéases. Cette augmentation se maintient dans le même rapport après séchage : on peut penser qu'une certaine germination des grains est responsable de ce fait dans le cas de l'échauffement. L'explication semble moins évidente lorsqu'il y a eu conservation par ventilation d'air refroidi, mais elle peut être recherchée vraisemblablement dans le développement des moisissures qui ont été nettement observées. 
- 1a disponibilité de la lysine apparaît nettement diminuée lorsque le séchage est effectué à haute température : cette baisse est plus accusée après l'échauffement en cellule : il faut remarquer cependant que la détermination par voie chimique ne rend pas forcément compte de la disponibilité nutritionnelle de la lysine. Dans un milieu aussi complexe que les céréales, plusieurs causes d'interférence peuvent intervenir et, en particulier, la haute teneur en glucides. Mais nous pensons toutefois que de telles variations sont l'indice de modifications profondes de la structure des protéines qui risquent fortement d'avoir des répercussions sur le plan nutritionnel en général, et particulièrement sur l'utilisation de la lysine.

\section{CONCLUSIONS}

Des résultats précédents, il ressort que le préstockage qui occasionne les plus importantes modifications des protéines est la conservation par ventilation d'air refroidi. In effet, on note, après celle-ci, une sensibilité accrue au traitement thermique qui se manifeste par une baisse de solubilité dès $80^{\circ} \mathrm{C}$, se répercutant quelque peu sur la teneur en lysine disponible; la digestion trypsique est la plus faible; l'activité protéolytique est élevée. Cette dernière, quelle que soit son origine, est vraisemblablement responsable de la légère augmentation de la fraction alcoolosoluble de l'échantillon $F_{0}$ et, peut-être, de la plus grande sensibilité thermique, par suite de modifications de structure dans les grosses molécules protéiques.

Le prétraitement par échauffement en cellules occasionne une évolution semblable, mais plus limitée, de l'activité protéolytique. La structure des protéines semble cependant moins modifiée : en effet, les résultats, aussi bien de la solubilité alcoolique que de la digestibilité trypsique, montrent, après les différentes opérations de séchage, des variations comparables à celles observées dans le cas de traitements thermiques effectués immédiatement après la récolte, bien que la disponibilité de la lysine soit autant affectée que dans le cas précédent.

\section{SUMMARY}

\section{V. - PROPERTIES OF PROTEINS}

The study of the evolution of proteins during wet-maize preliminary storage and drying has been undertaken to try to explain variations of the quality of grain. In the first place, the state of these components is influenced by the drying temperature, but its effect is more or less important according to the pretreatment undergone by the grain : so, a 45 days preservation by cooled air flow produces more important modifications than a 6 days spontaneous heating.

\section{RÉFÉRENCES BIBLIOGRAPHIQUES}

American Association of Cereal Chemist (A. A. C. C.). Ceveal Laboratory Methods. Method 22-60, 550 p. CNERNA Cahiers techniques $1958:$ Les méthodes analytiques des Céréales, 1, 423.

Busson F., Fauconneau G., Pion R., Montreuil J., ig66. Influence des conditions de production, de récolte et de stockage sur la composition biochimique des céréales ; répercussions des variations sur la valeur nutritionnelle $: 2^{\circ}$ section : acides aminés. Ann. Nutr. Alim., 20, 199-2 I9.

Carpenter K. J., r960. The estimation of the available lysine in animal protein foods. Biochem. J., YY7, 604-610. 